\title{
ESTIGMA INTERNALIZADO DE INDIVÍDUOS EM TRATAMENTO PARA DEPENDÊNCIA QUÍMICA E SUA RELAÇÃO COM A PRÁTICA DE ATIVIDADE FÍSICA
}

\author{
STIGMA INTERNALIZED BY INDIVIDUALS IN TREATMENT FOR SUBSTANCE \\ DEPENDENCE AND ITS RELATIONSHIP WITH PHYSICAL ACTIVITY
}

ESTIGMA INTERNALIZADO DE INDIVIDUOS EN TRATAMIENTO PARA DEPENDENCIA QUÍMICA Y SU RELACIÓN CON LA PRÁCTICA DE ACTIVIDAD FÍSICA

\section{Bruno Marson Malagodi*, Márcia Greguol*, Attilio Carraro**, Hélio Serassuelo Junior*}

\section{Palavras chave:}

Transtornos relacionados ao uso de substâncias. Estigma social. Alcoolismo. Exercício.
Keywords: Substance-related disorders. Social stigma. Alcoholism. Exercise.

Palabras clave: Trastornos relacionados al uso de substancias. Estigma social. Alcoholismo. Ejercicio.
Resumo: Indivíduos com dependência química tendem a ser estigmatizados e, uma vez que este estigma é internalizado, podem ocorrer prejuízos na adesão ao tratamento e a reinserção social. 0 objetivo do estudo é analisar o estigma internalizado de indivíduos em tratamento para dependência química e sua relação com a prática de atividade física. Para tanto, 106 indivíduos com dependência química responderam a questionários sobre estigma internalizado e prática de atividade física. Os dados foram tratados com estatística descritiva e análises de correlação. 0 estigma internalizado mostrou-se em geral elevado, com maiores pontuações para Alienação, Percepção de discriminação e Evitação social. Foi verificada correlação significativa inversa entre a prática de atividade física e o estigma internalizado, indicando que a oferta de programas de atividade física na fase de reabilitação pode ser benéfica não apenas para a melhora da condição física, mas também para a redução dos danos psicológicos impostos pela dependência química.

Abstract: Substance-dependent individuals tend to be stigmatized. Internalizing that stigma may result in impairments to their adherence to treatment and social reintegration. This study analyzed stigma internalized by individuals in treatment for substance dependence and its relation to physical activity. Questionnaires on internalized stigma and physical activity were responded by 106 substance-dependent individuals. The data were treated by descriptive statistics and correlation analyzes. Internalized stigma was generally high, with higher scores for Alienation, Perception of Discrimination, and Social Avoidance. Significant inverse correlation was found between physical activity and internalized stigma, indicating that physical activity programs during rehabilitation may not only improve those individuals' physical condition but also reduce the psychological damages imposed by substance dependency.

Resumen: Los individuos con dependencia química tienden a ser estigmatizados y, una vez que este estigma es internalizado, puede perjudicar la adhesión al tratamiento y la reinserción social. El objetivo del estudio es analizar el estigma internalizado de individuos en tratamiento para adicción química y su relación con la práctica de actividad física. Para ello, 106 individuos con dependencia química respondieron a cuestionarios sobre estigma internalizado y práctica de actividad física. Los datos fueron tratados con estadística descriptiva y análisis de correlación. El estigma internalizado se mostró en general elevado, con mayores puntuaciones para Alienación, Percepción de discriminación y Evitación social. Se verificó una correlación significativa inversa entre la práctica de actividad física y el estigma internalizado, indicando que la oferta de programas de actividad física en la fase de rehabilitación puede ser beneficiosa no solo para mejorar la condición física, sino también para la reducción de los daños psicológicos impuestos por la dependencia química.
*Universidade Estadual de Londrina. Londrina, PR, Brasil.

E-mail: brunomarson32@gmail.com; mgreguol@gmail.com; helioj@uel.b

**Universidade de Padova. Padova tália.

E-mail: attilio.carraro@unipd.it

Recebido em: 19-07-2018

Aprovado em: 20-05-2019

Publicado em: 06-09-2019

DOI

https://doi.org/10.22456/1982-8918.84970

(c) (i) () Licence 


\section{INTRODUÇÃO}

O consumo de álcool, tabaco e drogas ilícitas entre jovens e adultos representa um grande problema de saúde pública em todo o mundo e vem contribuindo de maneira significativa para o aumento da carga global de doenças e da violência (WHO, 2017). Estimase que 250 milhões de pessoas entre 15 e 64 anos de idade, ou seja, algo em torno de 5\% da população adulta global, tenha usado drogas pelo menos uma vez no ano de 2016. Ainda mais preocupante é o fato de que cerca de 29,5 milhões de pessoas, ou $0,6 \%$ da população adulta global, podem sofrer de transtornos relacionados ao uso abusivo de drogas (UNODC, 2017). Esse abuso é definido como um agrupamento de sintomas cognitivos, comportamentais e fisiológicos, indicando o uso contínuo da substância pelo indivíduo, apesar de significativos problemas advindos a partir deste comportamento (APA, 2014).

Intervenções baseadas em práticas de atividade física são consideradas abordagens potencialmente seguras, de baixo custo e não farmacológicas e podem ser utilizadas tanto nas fases iniciais quanto nos estágios posteriores do processo de tratamento de dependência química. Além disso, complementam abordagens tradicionais e apresentam benefícios secundários em termos da saúde geral dos indivíduos (por exemplo: prevenção de obesidade e de outras doenças crônicas, como a diabetes e a hipertensão arterial). A atividade física pode também ser efetiva como forma de prevenção de recaídas, devido aos efeitos positivos fisiológicos e psicológicos em pacientes que procuram ajuda para o tratamento da dependência química (CARRARO, 2018; KAUR, GARNAWAT, BHATIA, 2013; LYNCH et al., 2013).

Além dos fatores ligados aos aspectos físicos, especula-se que a prática de atividade física possa também ter influência em diversas variáveis psicológicas e sociais, melhorando a adesão ao tratamento, influenciando na aceitação e na mudança benéfica de comportamento (SCADUTO; BARBIERI, 2009). Uma variável que poderia ser influenciada pela prática de atividade física seria o estigma internalizado. 0 processo de estigmatização refere-se à desvalorização, perda de status e consequente discriminação de um indivíduo desencadeada pela atribuição de estereótipos negativos com base em características físicas e pessoais que ele possui, as quais são consideradas socialmente inaceitáveis (FELICISSIMO et al., 2013).

Segundo Soares et al. (2015), no que se refere às consequências do estigma para os indivíduos nessa condição, o principal impacto é sua internalização, fenômeno denominado estigma internalizado ou autoestigma. A internalização do estigma ocorre à medida que 0 indivíduo se torna consciente dos estereótipos negativos que as outras pessoas endossam (consciência dos estereótipos), concorda pessoalmente com esses estereótipos (concordância com estereótipos) e aplica esses estereótipos a si mesmo. 0 processo de internalização do estigma torna-se central para as condições psicológicas desses indivíduos, gerando diminuição de autoestima, autoconfiança e autoeficácia, percepção de descrédito, sentimentos de vergonha, culpa, angústia, raiva ou autorreprovação, assim como várias implicações práticas em sua vida (SOARES, 2011).

Dentre as condições de saúde que podem ser agravadas pelo estigma internalizado, a dependência de álcool e outras drogas tem uma relevância significativa, uma vez que é um dos principais transtornos estigmatizados em todo o mundo, sendo a moralização do consumo um dos principais aspectos envolvidos nesse processo. Existem também perspectivas limitadas de recuperação, consequentemente reforçando o isolamento social do indivíduo e a hesitação para a busca de ajuda profissional e tratamento adequado para sua condição (FELICISSIMO et al., 2013; SOARES et al., 2015). 
Devido à escassez de estudos na área e tendo em vista a relevância do tema, o presente estudo tem como objetivo analisar o estigma internalizado de indivíduos internados para o tratamento de dependência química, bem como sua possível relação com a prática da atividade física.

\section{MÉTODO}

O estudo foi do tipo descritivo correlacional e contou com a participação de 106 homens com idades entre 20 e 55 anos, submetidos ao processo terapêutico de internação voluntária para 0 tratamento de dependência química. Os pacientes eram provenientes de três instituições terapêuticas localizadas na cidade de Londrina/PR, as quais atendiam apenas pacientes do sexo masculino. Foram incluídos na amostra apenas os indivíduos que estavam na fase inicial do tratamento, na primeira ou segunda semana de ingresso na comunidade terapêutica. Como critério de exclusão, não puderam participar os pacientes que apresentavam comprometimentos cognitivos que dificultassem a compreensão dos instrumentos de avaliação (Miniexame do Estado Mental < 21 pontos).

Todos os participantes assinaram um Termo de Consentimento Livre e Esclarecido concordando em participar da pesquisa, e o estudo foi aprovado pelo Comitê de Ética em Pesquisa com Seres Humanos da Universidade Estadual de Londrina - UEL (Parecer: 2.125.747/2017).

Todos os questionários foram aplicados no mesmo dia, em horário agendado com cada participante e, após explicações preliminares, foram respondidos pelo indivíduo sem a interferência do pesquisador. Foi inicialmente aplicada uma anamnese composta por perguntas sobre idade, escolaridade, início do tratamento, se o indivíduo já foi ou não submetido a tratamentos anteriores, tipo de substância consumida, tempo de consumo e outras condições de saúde. Em seguida, os participantes do estudo responderam ao Questionário Baecke de Atividade Física Habitual (BAECKE; BUREMA; FRIJTERS, 1982), o qual já foi traduzido e adaptado para a língua portuguesa e utilizado em estudos no Brasil (FLORINDO; LATORRE, 2003). Esse questionário consiste de 16 questões em forma de Escala Likert de 1 a 5, envolvendo três escores relacionados aos 12 meses precedentes: (1) atividade física ocupacional, consistindo de oito questões, (2) esportes/exercícios físicos no lazer (consistindo de quatro questões), e (3) atividades de lazer e de locomoção (consistindo de quatro questões). O escore total para a atividade física habitual é obtido somando-se atividade física ocupacional + esportes/exercícios físicos no lazer + atividades de lazer e de locomoção.

Para avaliar o estigma internalizado, foi utilizada uma escala denominada ISMI-BR Versão Brasileira da Escala de Estigma Internalizado de Transtorno Mental adaptada para Dependentes de Substâncias, composta por 29 itens de uma escala do tipo Likert de 4 pontos (1 a 4) que varia de "discordo totalmente" a "concordo totalmente", traduzida e adaptada para dependentes de substâncias. A escala é dividida em cinco subitens: Alienação (7 questões - mede a experiência subjetiva de ser avaliado de forma inferior aos demais, a sensação de "não pertencimento" ou de ter a identidade deteriorada); Aprovação do estereótipo (6 questões - mede o grau de concordância dos pacientes com os estereótipos sobre as pessoas com dependência química); Percepção de discriminação (5 questões - identifica a percepção dos pacientes sobre a maneira como são frequentemente tratados pelos outros); Evitação social (6 questões - identifica estratégias de evitação das relações sociais) e Resistência ao estigma (5 
questões - descreve a experiência de resistir ou não ser afetado pelo estigma internalizado). Neste último item, a pontuação deve ser invertida para o cálculo do escore total do questionário, já que maior resistência ao estigma é um indicativo de menor estigma internalizado.

A pontuação total varia de 29 a 116, dessa forma, dividindo-se a pontuação total por 29 é possível obter o escore médio, que varia de 1 a 4 . Quanto maior a pontuação ou o escore mais elevado será o nível de estigma internalizado, ou seja, mais forte a internalização do estigma percebido pela sociedade sobre sua condição atual. Escores acima de 2,5 denotam elevado estigma internalizado (RITSHER; PHELAN, 2004). A escala é de fácil aplicação e apresenta bons índices de confiabilidade e validade (RITSHER; OTILINGA; GRAJALES, 2003; SOARES, 2011).

Após a verificação da normalidade dos dados, foi utilizada estatística descritiva, com valores médios e de variabilidade para as variáveis contínuas e porcentagem de resposta para as variáveis categóricas. Para verificar possíveis relações entre o estigma internalizado, 0 tempo de uso de substâncias e a prática de atividade física, foi utilizado teste de Correlação de Pearson. Para a comparação dos indivíduos por grupos etários (distribuição da idade em quartis), por tempo de uso (distribuição do tempo de uso em quartis), tipo de droga consumida (álcool, cocaína/crack ou ambos) e por escolaridade (fundamental completo e incompleto, médio completo e incompleto e superior completo e incompleto), foi realizada Análise de Variância (ANOVA). A significância adotada foi $p \leq 0,05$.

\section{RESULTADOS}

Os dados descritivos dos participantes do estudo encontram-se sintetizados na Tabela 1.

Tabela 1- Dados descritivos dos participantes do estudo

\begin{tabular}{lcc}
\hline Variáveis & Média & Desvio Padrão \\
\hline Idade (anos) & 35,39 & 11,0 \\
Tempo de uso de substâncias (anos) & 13,99 & 10,3 \\
Pontuação total ISMI & 76,76 & 9,7 \\
Escore ISMI & 2,65 & 0,3 \\
Alienação & 2,9 & 0,5 \\
Aprovação do estereótipo & 2,42 & 0,5 \\
Percepção de discriminação & 2,72 & 0,5 \\
Evitação social & 2,61 & 0,5 \\
Resistência ao estigma & 2,37 & 0,5 \\
Escore Atividade Física Ocupacional & 2,34 & 0,3 \\
Escore Esporte/Exercícios no Lazer & 2,32 & 0,3 \\
Escore Atividades de Lazer /Locomoção & 2,73 & 0,3 \\
Escore total Baecke & 7,39 & 0,7 \\
\hline
\end{tabular}

Fonte: dados da pesquisa

Quando questionados sobre a escolaridade, a prevalência foi de nível mais baixo, com $53,8 \%(n=57)$ dos participantes relatando ter nível fundamental completo ou incompleto e apenas 4,7\% ( $n=5)$ declarando ter concluído o ensino superior. Sobre o tipo de substância de abuso, 27,4\% ( $n=29)$ afirmaram consumir apenas álcool, 25,5\% $(n=27)$ crack e cocaína e $46,2 \%(n=49)$ declaram consumir drogas e álcool. 
De acordo com os resultados do ISMI, $68,9 \%$ dos participantes do estudo ( $n=73$ ) assinalaram escore acima de 2,5, indicando elevado estigma internalizado. Os subitens com maiores escores foram a Alienação, seguida da Percepção de discriminação e Evitação social, todos acima de 2,5 pontos.

Nas comparações entre os grupos etários, os indivíduos alocados no primeiro quartil (até 25 anos) apresentaram valores significativamente mais baixos na Evitação social ( $p=$ $0,02)$ em comparação aos indivíduos mais velhos. Já nas comparações entre os diversos níveis de escolaridade, foi observado que indivíduos com escolaridade mais elevada (nível superior completo ou incompleto) também exibiram menores valores de Evitação social $(p=0,04)$ do que aqueles com menor escolaridade. Já as comparações entre os tipos de substâncias consumidas e entre os quartis por tempo de uso não resultaram em diferenças significativas em nenhuma das variáveis.

Uma última análise comparativa foi realizada entre os indivíduos que apresentaram escore total no ISMI de até 2,5 (resultado considerado não elevado) e maior do que 2,5 (resultado considerado elevado) com relação aos indicadores de atividade física e foram observadas diferenças significativas em todos os domínios do Baecke, bem como no escore total $(p \leq 0,01)$, indicando que indivíduos que exibiram menores valores de estigma internalizado realizaram uma quantidade maior de atividade física no ano precedente à internação do que aqueles com estigma elevado.

No que se refere às análises de correlação entre os escores da atividade física nos últimos 12 meses e do estigma internalizado, os resultados indicaram relações significativas, discriminadas na Tabela 2. Podem ser observadas correlações significativas e negativas entre os escores parciais e total do questionário de estigma internalizado (ISMI), com exceção da variável Resistência ao estigma, e os escores por domínio e total do questionário Baecke, com mais força para a atividade física ocupacional e o esporte/exercícios físicos no lazer. Estes dados indicam que, quanto maior a prática de atividade física nos 12 meses precedentes à internação, menores os valores manifestados de estigma internalizado. Não foram verificadas correlações significativas entre o estigma internalizado e a idade ou o tempo de uso de substâncias.

Tabela 2- Correlações entre os escores dos questionários de atividade física e de estigma internalizado

\begin{tabular}{|c|c|c|c|c|c|}
\hline \multicolumn{2}{|c|}{ Itens do questionário } & $\begin{array}{c}\text { Atividade Física } \\
\text { Ocupacional }\end{array}$ & $\begin{array}{c}\text { Esporte/Exercícios } \\
\text { no Lazer }\end{array}$ & $\begin{array}{c}\text { Atividades de Lazer/ } \\
\text { Locomoção }\end{array}$ & Baecke total \\
\hline \multirow{2}{*}{ Alienação } & $r$ & $-0,48^{*}$ & $-0,55^{\star}$ & $-0,36^{*}$ & $-0,56^{*}$ \\
\hline & $p$ & 0,00 & 0,00 & 0,00 & 0,00 \\
\hline \multirow{2}{*}{$\begin{array}{l}\text { Aprovação do } \\
\text { estereótipo }\end{array}$} & $r$ & $-0,62^{*}$ & $-0,61^{*}$ & $-0,27^{\star}$ & $-0,61^{*}$ \\
\hline & $p$ & 0,00 & 0,00 & 0,01 & 0,00 \\
\hline \multirow{2}{*}{$\begin{array}{l}\text { Percepção de } \\
\text { discriminação }\end{array}$} & $r$ & $-0,45^{\star}$ & $-0,49^{\star}$ & $-0,29^{*}$ & $-0,5^{\star}$ \\
\hline & $p$ & 0,00 & 0,00 & 0,01 & 0,002 \\
\hline \multirow{2}{*}{ Evitação social } & $r$ & $-0,56^{\star}$ & $-0,66^{*}$ & $-0,36^{*}$ & $-0,64^{*}$ \\
\hline & $p$ & 0,00 & 0,00 & 0,00 & 0,00 \\
\hline \multirow{2}{*}{$\begin{array}{l}\text { Resistência ao } \\
\text { estigma }\end{array}$} & $r$ & 0,04 & 0,03 & 0,14 & 0,08 \\
\hline & $p$ & 0,65 & 0,8 & 0,16 & 0,39 \\
\hline \multirow{2}{*}{ ISMI total } & $r$ & $-0,67^{\star}$ & $-0,72^{*}$ & $-0,43^{\star}$ & $-0,74^{*}$ \\
\hline & $p$ & 0,00 & 0,00 & 0,00 & 0,00 \\
\hline
\end{tabular}




\section{DISCUSSÃO}

O objetivo do estudo foi investigar o estigma internalizado de indivíduos internados para tratamento de dependência química e sua possível relação com a prática de atividade física. Entre os participantes, foi observada maior porcentagem de indivíduos com baixa escolaridade e com tempo de dependência superior a dez anos. Segundo a Organização Mundial da Saúde (WHO, 2017) o início do consumo de álcool, cigarro e outras drogas ocorre predominantemente durante a adolescência, o que pode justificar o fato de a maior parte dos indivíduos consumirem o álcool e outras substâncias por um logo período antes de buscarem tratamento. Ainda, como em muitos casos o início do consumo de substâncias é precoce e a doença se instala de maneira progressiva, geralmente ocorre o desinteresse e consequente abandono escolar em idades mais jovens, o que explicaria a baixa escolaridade observada (CARDOSO; MALBERGIER, 2014; MARTINS; PILLON, 2008).

Nas análises dos escores relativos à prática habitual de atividade física pelo questionário Baecke, os valores dos domínios Esporte/Exercícios no Lazer e Atividades de Lazer e Locomoção, assim como o escore médio total, foram semelhantes aos de outros estudos envolvendo populações portadoras de outras doenças crônicas (FLORINDO et al., 2006; TEHARD et al., 2005). Já os resultados obtidos no domínio das Atividades Ocupacionais foram inferiores, o que pode ter ocorrido por conta das consequências advindas do consumo de substâncias, as quais, em algumas situações, levam à necessidade de afastamento parcial ou até total do indivíduo de suas atividades ocupacionais (LI et al., 2017).

Quanto aos resultados obtidos na aplicação do ISMI, a maior parte dos indivíduos reportou elevados níveis de estigma internalizado $(61,7 \%)$, com maior intensidade para os itens Alienação, Percepção de discriminação e Evitação social. Assim, entre os indivíduos com dependência química, a internalização do estigma parece ocorrer com maior evidência na sensação de não se enquadrar de maneira adequada na sociedade, na forma como percebem que são discriminados pelas demais pessoas e na tendência de evitar relações sociais. Tais fatos devem ser vistos com atenção pela equipe de reabilitação, uma vez que podem gerar um impacto negativo na adesão ao tratamento, na qualidade de vida e no processo de reintegração social (SIBITZ et al., 2013).

Estudo realizado por Ahern, Stuber e Galea (2007) com usuários de drogas nos Estados Unidos mostrou que $85 \%$ deles relataram que muitas pessoas os percebem como pouco confiáveis ou perigosos. Observou-se ainda que os usuários de drogas evitavam o contato com outras pessoas por se sentires inferiores, e que as discriminações mais percebidas foram entre familiares e amigos. Ressalta-se que 0 efeito dessas crenças disfuncionais e 0 aumento do sentimento de inferioridade geram emoções negativas, ansiedade, depressão, angústia, vergonha ou culpa, levando ao isolamento social, à redução da autoestima e da autoeficácia e comprometendo a crença que o indivíduo tem no sucesso do tratamento.

Os valores médios do estigma internalizado obtidos no presente estudo são superiores a outros que analisaram pacientes com esquizofrenia (LI et al., 2017; MARGETIC et al., 2010; TANABE; HAYASHI; IDENO, 2016) e outras doenças psiquiátricas (LIEN et al., 2015), provavelmente pelo fato de a dependência química ainda ser vista por muitas pessoas não como uma doença, mas sim como um comportamento inadequado do indivíduo, produto de hábitos disfuncionais ou falta de caráter (RONZANI; NOTO; SILVEIRA, 2014). Além disso, 
indivíduos com dependência química são vistos socialmente como perigosos, violentos e imprevisíveis, o que acaba por restringir oportunidades e redes sociais, uma vez que antecipam a rejeição e evocam sentimentos de vergonha, culpa e percepção de descrédito. No caso da dependência química e outros distúrbios psiquiátricos, há uma maior probabilidade de que seus comportamentos atípicos ou desviantes das normas sociais tenham sua causa atribuída a fatores próprios do indivíduo, como falta de força de vontade (LYSAKER et al., 2007; RONZANI; NOTO; SILVEIRA, 2014).

O estigma internalizado tem sido considerado por alguns autores como um elemento que gera um estereótipo, separação, perda de status e discriminação, associando-se a uma qualidade de vida precária, baixa autoestima, isolamento e degradação das relações sociais (LI et al., 2017; RONZANI; NOTO; SILVEIRA, 2014; TANABE; HAYASHI; IDENO, 2016). Para pessoas com dependência química, altos níveis de estigma internalizado podem gerar baixa adesão ao tratamento e, consequentemente, perspectivas limitadas de recuperação, agravando quadros depressivos, reduzindo as participações em atividades sociais e a busca por serviços de saúde (CAMA et al., 2016). Ainda Cama et al. (2016), analisando o estigma internalizado em usuários de drogas injetáveis, verificaram que este se associava com depressão prévia e maior gravidade da dependência. Os autores destacaram ainda que o estigma internalizado se relaciona a problemas com o funcionamento comportamental e maior predisposição ao suicídio.

Os resultados do presente estudo indicaram correlações inversas significativas entre 0 estigma internalizado e a prática de atividade física, com mais força nos escores relacionados às atividades ocupacionais e exercícios/esportes no lazer. Estes dados indicam que, quanto mais atividade física havia sido praticada no ano anterior, menores valores eram reportados de estigma internalizado pelo indivíduo. Uma possível explicação para tais associações residiria no fato que a prática de atividade física pode impactar de maneira positiva 0 autoconceito e autoimagem do indivíduo, o que influenciaria a forma como ele percebe e internaliza 0 estigma da dependência química (HORSSELEMBERG et al., 2016; RODRIGUEZ; AUDRAINMcGOVERN, 2005).

Quando verificadas as subescalas do ISMI, a correlação mais forte foi entre a prática total de atividade física e a Evitação social, mostrando que indivíduos que praticaram menor quantidade total de atividade física no ano precedente também buscam evitar com mais força as relações de convívio social. Resultado semelhante foi visto por Moriarty et al. (2012), que observaram relações inversas significativas entre a Evitação social e a realização de atividades entre indivíduos com distúrbios psiquiátricos. No entanto, conforme os autores destacam, não é possível estabelecer uma relação causal, visto que tanto a Evitação social pode reduzir as oportunidades de prática de atividade física quanto o contrário.

Alguns autores têm apontado que existe uma relação inversa entre o estigma internalizado e a autoestima, embora não seja possível identificar claramente uma relação de causalidade (FELICISSIMO et al., 2013; HORSSELEMBERG et al., 2016). Oliveira, Carvalho e Esteves (2016), analisando pacientes com doenças mentais, verificaram que a autoestima é um elemento central para a redução dos efeitos negativos do estigma internalizado na qualidade de vida e isso seria mais evidente em pessoas que possuíam um autoconceito físico mais elevado. Esses fatos parecem corroborar a ideia de que, ao fortalecer a autoestima e o autoconceito físico do indivíduo, seria possível amenizar as consequências negativas do estigma internalizado. 
Alguns estudos já têm destacado que programas de atividade física, especialmente associados ao apoio de equipe multiprofissional, podem ter efeitos terapêuticos benéficos na melhora do autoconceito e da autoestima e na consequente redução do estigma internalizado em indivíduos com esquizofrenia (MORIARTY et al., 2012; SIBITZ et al., 2013; VANCAMPFORT et al., 2011), epilepsia (TEDRUS; STERCA; PEREIRA, 2017) e distúrbios alimentares (MENSINGER; MEADOWS, 2017; VANCAMPFORT et al., 2014). Para pacientes com dependência química, a prática de atividades físicas também tem se mostrado relevante para a melhora de quadros depressivos, da adesão ao tratamento, da prevenção de recaídas e do autoconceito global (ZSCHUCKE; HEINZ; STROHLE, 2012). Assim, tendo em vista a elevada associação inversa observada entre a prática de atividade física e o estigma internalizado no presente estudo, especula-se que essa possa ser uma estratégia relevante no processo terapêutico de indivíduos em tratamento para dependência química.

Conforme mencionado anteriormente, a redução do estigma internalizado é fundamental para a melhora da qualidade de vida e da reinserção social do paciente com dependência química (RONZANI et al., 2014). Para que isso ocorra, no entanto, devem ser focadas estratégias que atuem na sociedade e no próprio indivíduo. Com relação à sociedade, a educação, a divulgação de informações sobre a doença e o contato podem contribuir para a quebra dos estereótipos (RONZANI; NOTO; SILVEIRA, 2014). Já no que tange ao indivíduo, o processo terapêutico deve focar no ganho de autonomia, empoderamento e fortalecimento da autoestima (SIBITZ et al., 2013).

Há de se destacar, no entanto, que os escores referentes à prática de atividade física foram baixos quando comparados aos de homens adultos saudáveis (OLIVEIRA et al., 2012; FLORINDO; LATORRE, 2003), especialmente no tocante às práticas de esporte e exercícios no lazer, justamente aquelas que apresentaram relação inversa mais forte com o estigma internalizado. Assim, ressalta-se a importância de que sejam oferecidas mais oportunidades de práticas de atividade física para indivíduos em tratamento para dependência química, não apenas pelos potenciais benefícios físicos, mas também pela possibilidade dessa prática aprimorar aspectos psicológicos, reforçando a crença do paciente em si mesmo e tornando mais positiva sua percepção sobre o tratamento e sua posterior reinserção social.

\section{CONCLUSÃO}

Os resultados observados no estudo permitem supor uma associação inversa entre a prática de atividade física e o estigma internalizado de indivíduos em tratamento para dependência química. A maior parte dos participantes do estudo mostrou elevados valores de estigma internalizado, sobretudo nos itens referentes à Alienação, Percepção de discriminação e Evitação social, e níveis mais baixos de atividade física no que se refere à prática de esporte e exercícios no lazer.

Sugere-se que estudos sejam conduzidos envolvendo intervenções com programas de exercícios físicos direcionados para indivíduos com dependência química, de modo que seja possível estabelecer relação de causa-efeito com o estigma internalizado. Reforça-se também a importância da oferta de programas de exercícios físicos sistematizados e ministrados por profissionais da área da Educação Física dentro da abordagem multiprofissional em clínicas e comunidades terapêuticas, como forma de melhorar a adesão ao tratamento e aprimoramento 
das capacidades físicas e psicológicas do indivíduo que busca a recuperação inicial e manutenção do seu estado de saúde e qualidade de vida.

\section{REFERÊNCIAS}

AHERN, Jennifer; STUBER, Jennifer; GALEA, Sandro. Stigma, discrimination and the health of illicit drug users. Drug and alcohol dependence, v. 88, n. 2-3, p. 188-196, 2007.

APA - AMERICAN PSYCHIATRIC ASSOCIATION. DSM-5: manual diagnóstico e estatístico de transtornos mentais. 5. ed. Porto Alegre: Artmed, 2014. p. 483 - 487.

BAECKE, Jos A.H.; BUREMA, Jan; FRIJTERS, Jan E. A short questionnaire for the measurement of habitual physical activity in epidemiological studies. The American journal of clinical nutrition, v. 36, n. 5, p. 936-942, 1982.

CAMA, Elena et al. Internalized stigma among people who inject drugs. Substance use \& misuse, v. 51, n. 12, p. 1664-1668, 2016.

CARDOSO, Luciana Roberta Donola; MALBERGIER, André. Problemas escolares e o consumo de álcool e outras drogas entre adolescentes. Psicologia Escolar e Educacional, v. 18, n. 1, p. 27-34, 2014.

CARRARO, Attilio. Atividade física e saúde mental. In: GREGUOL, Márcia; COSTA, Roberto Fernandes. Atividade Física Adaptada e Saúde: qualidade de vida para pessoas com necessidades especiais. Barueri: Manole, 2018. p.410-433.

FELICISSIMO, Flaviane Bevilaqua et al. Estigma internalizado e autoestima: uma revisão sistemática da literatura. Revista Psicologia-Teoria e Prática, v. 15, n. 1, p. 116-129, 2013.

FLORINDO, Alex Antonio; LATORRE, Maria do Rosario D. O. Validation and reliability of the Baecke questionnaire for the evaluation of habitual physical activity in adult men. Revista Brasileira de Medicina do Esporte, v. 9, n. 3, p. 129-135, 2003.

FLORINDO, Alex Antonio et al. Validity and reliability of the Baecke questionnaire for the evaluation of habitual physical activity among people living with HIV/AIDS. Cadernos de saúde pública, v. 22, n. 3, p. 535-541, 2006.

HORSSELENBERG, Ellen MA et al. Self-Stigma and Its Relationship with Victimization, Psychotic Symptoms and Self-Esteem among People with Schizophrenia Spectrum Disorders. PloS one, v. 11, n. 10, p. e0149763, 2016.

KAUR, Jaswinder; GARNAWAT, Deepti; BHATIA, M. S. Psychophysiotherapy. Rehabilitation for substance abuse disorders. Delhi Psychiatry Journal, v. 16, n. 2, p. 400-403, 2013.

LI, Jie et al. Stigma and discrimination experienced by people with schizophrenia living in the community in Guangzhou, China. Psychiatry Research, v. 255, p. 225-231, 2017.

LIEN, Yin-Ju et al. Internalized stigma and stigma resistance among patients with mental illness in Han Chinese population. Psychiatric Quarterly, v. 86, n. 2, p. 181-197, 2015.

LYNCH, Wendy J. et al. Exercise as a novel treatment for drug addiction: a neurobiological and stagedependent hypothesis. Neuroscience \& Biobehavioral Reviews, v. 37, n. 8, p. 1622-1644, 2013. 
LYSAKER, Paul Henry et al. Clinical and psychological correlates of two domains of hopelessness in schizophrenia. Journal of Rehabilitation Research and Development, v. 45, n. 6, p. 911-919, 2008.

MARGETIĆ, Branka Aukst et al. Relations of internalized stigma with temperament and character in patients with schizophrenia. Comprehensive Psychiatry, v. 51, n. 6, p. 603-606, 2010.

MARTINS, Mayra Costa; PILLON, Sandra Cristina. A relação entre a iniciação do uso de drogas e o primeiro ato infracional entre os adolescentes em conflito com a lei. Cadernos de Saúde Pública, v. 24, p. 1112-1120, 2008.

MENSINGER, Janell L.; MEADOWS, Angela. Internalized weight stigma mediates and moderates physical activity outcomes during a healthy living program for women with high body mass index. Psychology of Sport and Exercise, v. 30, p. 64-72, 2017.

MORIARTY, Anna et al. Understanding reduced activity in psychosis: the roles of stigma and illness appraisals. Social psychiatry and psychiatric epidemiology, v. 47, n. 10, p. 1685-1693, 2012.

OLIVEIRA, Sandra; CARVALHO, Helena; ESTEVES, Francisco. Internalized stigma and quality of life domains among people with mental illness: the mediating role of self-esteem. Journal of Mental Health, v. 25, n. 1, p. 55-61, 2016.

OLIVEIRA, Tiago Peçanha et al. Influência do nível de atividade física, na ocorrência de hipotensão pós-exercício em indivíduos normotensos. HU Revista, v. 37, n. 2, p.199-205, 2012.

RITSHER, Jennifer Boyd; OTILINGAM, Poorni G.; GRAJALES, Monica. Internalized stigma of mental illness: psychometric properties of a new measure. Psychiatry research, v. 121, n. 1, p. 31-49, 2003.

RITSHER, Jennifer Boyd; PHELAN, Jo C. Internalized stigma predicts erosion of morale among psychiatric outpatients. Psychiatry research, v. 129, n. 3, p. 257-265, 2004.

RODRIGUEZ, Daniel; AUDRAIN-MCGOVERN, Janet. Physical activity, global physical self-concept, and adolescent smoking. Annals of Behavioral Medicine, v. 30, n. 3, p. 251-259, 2005.

RONZANI, Telmo Mota; NOTO, Ana Regina; SILVEIRA, Pollyanna Santos da. Reduzindo o estigma entre usuários de drogas. Guia para profissionais e gestores. Juiz de Fora: Editora UFJF, 2014.

SCADUTO, Alessandro Antonio; BARBIERI, Valéria. O discurso sobre a adesão de adolescentes ao tratamento da dependência química em uma instituição de saúde pública. Ciências \& saúde coletiva, v.14, n.2, p.605-614, 2009.

SIBITZ, Ingrid et al. The impact of recovery-oriented day clinic treatment on internalized stigma: preliminary report. Psychiatry research, v. 209, n. 3, p. 326-332, 2013.

SOARES, Rhaisa Gontijo. Validação da versão brasileira da escala de estigma internalizado de transtorno mental (ISMI) adaptada para dependentes de substâncias. 2011. 105 f. Dissertação (Mestrado em Psicologia) - Universidade Federal de Juiz de Fora, Juiz de Fora, 2011.

SOARES, Rhaisa Gontijo et al. Validação da Versão Brasileira da Escala ISMI Adaptada para Dependentes de Substâncias. Psicologia: teoria e pesquisa, v. 31, n. 2, p. 229-238, 2015.

TANABE, Yosuke; HAYASHI, Kunihiko; IDENO, Yuki. The Internalized Stigma of Mental IIIness (ISMI) scale: validation of the Japanese version. BMC psychiatry, v. 16, n. 1, p. 116, 2016. 
TEDRUS, Gloria Maria Almeida Souza; STERCA, Guilherme Sabbag; PEREIRA, Renato Buarque. Physical activity, stigma, and quality of life in patients with epilepsy. Epilepsy \& Behavior, v. 77, p. 96-98, 2017.

TEHARD, Bertrand et al. Comparison of two physical activity questionnaires in obese subjects: the NUGENOB study. Medicine and science in sports and exercise, v. 37, n. 9, p. 1535-1541, 2005.

UNODC - UNITED NATIONS OFFICE ON DRUGS AND CRIME. World Drug Report 2017.

Disponível em: https://www.unodc.org/wdr2017/index.html. Acesso em: 30 jan.2018.

VANCAMPFORT, Davy et al. Physical activity participation, functional exercise capacity and selfesteem in patients with schizophrenia. International Journal of Therapy and Rehabilitation, v. 18, n. 4, p. 222-229, 2011.

VANCAMPFORT, Davy et al. Changes in physical activity, physical fitness, self-perception and quality of life following a 6-month physical activity counseling and cognitive behavioral therapy program in outpatients with binge eating disorder. Psychiatry research, v. 219, n. 2, p. 361-366, 2014.

WHO. WORLD HEALTH ORGANIZATION. Drug abuse. Disponível em: http://www.who.int/. Acesso em: 14 jun./2017.

ZSCHUCKE, Elisabeth; HEINZ, Andreas; STRÖHLE, Andreas. Exercise and physical activity in the therapy of substance use disorders. The Scientific World Journal, doi: 10.1100/2012/901741, may 2012. 